\title{
Compelling evidence of renormalons in QCD from high order perturbative expansions
}

\author{
Clemens Bauer, ${ }^{1}$ Gunnar S. Bali, ${ }^{1}$ and Antonio Pineda ${ }^{2}$ \\ ${ }^{1}$ Institut für Theoretische Physik, Universität Regensburg, D-93040 Regensburg, Germany \\ ${ }^{2}$ Grup de Física Teòrica, Universitat Autònoma de Barcelona, E-08193 Bellaterra, Barcelona, Spain
}

(Dated: September 13, 2018)

\begin{abstract}
We compute the static self-energy of SU(3) gauge theory in four spacetime dimensions to order $\alpha^{20}$ in the strong coupling constant $\alpha$. We employ lattice regularization to enable a numerical simulation within the framework of stochastic perturbation theory. We find perfect agreement with the factorial growth of high order coefficients predicted by the conjectured renormalon picture based on the operator product expansion.
\end{abstract}

PACS numbers: 11.15.Bt,12.38.Cy,12.38.Bx,11.10.Jj,12.39.Hg

Little is known about properties of quantum field theories from first principles. This is particularly so for asymptotically free gauge theories such as quantum gluodynamics. One of the most salient features of this theory is the confinement of charged objects. Yet this property has not been proven, and the best evidence comes from the linearly rising static potential at large distances obtained in lattice simulations. Another expected property is the asymptotic nature of perturbative weak coupling expansions. In four dimensional non-Abelian gauge theories one particular pattern of asymptotic divergence should be determined by the structure of the operator product expansion (OPE). It is usually named renormalon [1] or, more specifically, infrared renormalon. Its existence has also not been proven but only tested assuming the dominance of $\beta_{0}$-terms, which amounts to an effective Abelianization of the theory, or in the two dimensional $\mathrm{O}(N)$ model [2], where it is suppressed by powers of $1 / N$. Moreover, the possible non-existence or irrelevance of renormalons in Quantum Chromodynamics has been suggested in several papers, see, e.g. [3, 4] and references therein. This has motivated dedicated high order perturbative expansions of the plaquette, e.g. [58], in lattice regularization, with conflicting conclusions. Powers as high as $\alpha^{20}$ were achieved in the most recent simulation [9]. However, the expected asymptotic behaviour was not seen. If confirmed, this non-observation would cast doubt on the well-accepted lore of the OPE and renormalon physics (see [10] for a comprehensive review), and would significantly affect the phenomenological analysis of data from high energy physics experiments on the decay of heavy hadrons, heavy quark masses, the running coupling parameter, parton distributions, etc.. Therefore, this issue should be clarified unambiguously.

In this letter we present compelling numerical evidence that the expected renormalons indeed exist not only in models but in real gluodynamics. We also argue why previous analyses based on the plaquette have failed to detect them. The vital and new ingredients of our study are as follows.

(a) We consider a perturbative series whose leading renormalon is dictated by a dimension $d=1$ operator, rather than by the $d=4$ plaquette.

(b) Using a higher order integrator and employing twisted boundary conditions, among other improvements, we are able to obtain results of unprecedented precision on an extensive set of spacetime volumes.

(c) We carefully extrapolate to the infinite volume limit, thoroughly investigating finite size effects.

Perturbative expansions in powers of $\alpha$,

$$
K=\sum_{n} k_{n} \alpha^{n}
$$

are believed to be asymptotic and not Borel summable in QCD, due to the existence of singularities in the Borel plane (renormalons). Typically $k_{n}$ will diverge like $a_{d}^{n} n$ !, with a constant $a_{d}$. This divergence pattern of $k_{n}$ should not be arbitrary but consistent with the OPE associated to a physical observable. Even though this factorial growth was originally discovered analyzing the Feynman diagrams that contribute to the large $\beta_{0}$ approximation, the correct divergent structure can only be inferred by assuming that the perturbative series is asymptotic and complies with the OPE. The OPE fixes the positions and the structure of the renormalon singularities in the complex Borel plane, resulting in a more intricate pattern that cannot be obtained from the large $\beta_{0}$ approximation alone. Successive contributions $k_{n} \alpha^{n}$ decrease for small orders $n$ down to a minimum at $n_{0} \sim 1 /\left(\left|a_{d}\right| \alpha\right)$. Higher order contributions should be neglected and introduce an ambiguity of the order of this minimum term, $k_{n_{0}} \alpha^{n_{0}} \sim \exp \left[-1 /\left(\left|a_{d}\right| \alpha\right)\right]$.

Within the OPE an observable $R(q, \Lambda)$ can be factorized into short distance Wilson coefficients $C_{i}(q, \mu)$ and non-perturbative matrix elements $\left\langle O_{i}(\mu, \Lambda)\right\rangle$ of dimension $i$ :

$R=C_{0}(q, \mu)\left\langle O_{0}(\mu, \Lambda)\right\rangle+C_{d}(q, \mu)\left\langle O_{d}(\mu, \Lambda)\right\rangle\left(\frac{\Lambda}{q}\right)^{d}+\cdots$.

$q, \Lambda$ and $\mu$ denote a perturbative, low momentum and factorization scale, respectively, so that $q \gg \mu \gg \Lambda$. For the plaquette, $\left\langle O_{0}\right\rangle=1$ and the next higher nonvanishing operator is the dimension $d=4$ gluon condensate. In this case, the perturbative expansion of $C_{0}$ 
cannot be more accurate than $\mathcal{O}\left(\Lambda^{4} / q^{4}\right)$ which is exactly of the size of the $k_{n_{0}} \alpha^{n_{0}}$ term since

$$
\left(\frac{\Lambda}{q}\right)^{d} \simeq \exp \left(-\frac{1}{\left|a_{d}\right| \alpha}\right), \quad \text { where } \quad a_{d}=\frac{\beta_{0}}{2 \pi d},
$$

with $\beta_{0}=11$. The so-called leading infrared renormalon of this expansion cancels the ultraviolet ambiguity of the next order non-perturbative matrix element so that the physical observable $R$ is well-defined.

From this discussion it is evident that we should study series expansions with the smallest possible $n_{0}$ or, equivalently, $d$. For $d=1$ the perturbative expansion should start to diverge at an order $n_{0}$ that amounts to about one fourth of that for the plaquette. This applies to the pole mass (see [11, 12]) and to the associated self-energy of a static source, which we consider here. The latter does not have a continuum limit, as it linearly depends on the ultraviolet regulator. Here we consider lattice regularization with the Wilson gauge action [13] and write the self-energy in the following way:

$$
\delta m=\frac{1}{a} \sum_{n \geq 0} c_{n} \alpha^{n+1}(1 / a) .
$$

$a^{-1}$, the inverse lattice spacing, provides the ultraviolet cut-off. The large $n$ behaviour of the coefficients $c_{n}$ is regulator independent, universal and equal to the asymptotic behaviour of the pole mass up to $\mathcal{O}\left(e^{-1 / n}\right)$ terms (due to subleading renormalons):

$$
\begin{aligned}
c_{n} \stackrel{n \rightarrow \infty}{=} & N_{m}\left(\frac{\beta_{0}}{2 \pi}\right)^{n} \frac{\Gamma(n+1+b)}{\Gamma(1+b)} \\
& \times\left(1+\frac{b}{(n+b)} s_{1}+\cdots\right) .
\end{aligned}
$$

The coefficients $b$ and $s_{1}$ were computed in [14]. They read (see [15] for details)

$$
b=\frac{\beta_{1}}{2 \beta_{0}^{2}}, \quad s_{1}=\frac{1}{4 \beta_{0}^{3} b}\left(\frac{\beta_{1}^{2}}{\beta_{0}}-\beta_{2}\right) .
$$

For a static source in the fundamental (triplet) representation the normalization constant $N_{m}$ is exactly the same as for the leading renormalon of a heavy quark pole mass. This renormalon is also related to a renormalon of the singlet static potential since these contributions cancel from the energy $E(r)=2 m+V(r)$ [16 18]. For adjoint sources it corresponds to a specific combination of pole mass and adjoint static potential renormalons [19]. The factor $N_{m}$ is cancelled in the ratios

$$
\frac{c_{n}}{c_{n-1}} \frac{1}{n}=\frac{\beta_{0}}{2 \pi}\left[1+\frac{b}{n}-\left(1-b s_{1}\right) \frac{b s_{1}}{n^{2}}+\mathcal{O}\left(\frac{1}{n^{3}}\right)\right] \text {. }
$$

We obtain the expansion coefficients $c_{n}$ of the static energy from the temporal Polyakov line on hypercubic
TABLE I. Lattice geometries. Volumes with boldface time extents are expanded up to $\mathcal{O}\left(\alpha^{20}\right)$, the others up to $\mathcal{O}\left(\alpha^{12}\right)$.

\begin{tabular}{cccc}
\hline \hline$N_{S}$ & $N_{T}$ & $N_{S}$ & $N_{T}$ \\
\hline 7 & $\mathbf{7}, \mathbf{8}$ & 11 & $\mathbf{1 6}$ \\
8 & $\mathbf{8}, \mathbf{1 0}, 12,16$ & 12 & $\mathbf{1 2}$ \\
9 & $\mathbf{1 2}$ & 14 & $\mathbf{1 4}$ \\
10 & $8, \mathbf{1 0}, 12,16,20$ & 16 & $12,16,20$ \\
\hline \hline
\end{tabular}

lattices. We investigate volumes of $N_{T}$ lattice points in the time direction and spatial extents of $N_{S}$ points. Formally we may introduce an anisotropy $a_{t} \neq a_{s}$. In this case the lattice action, that is invariant under time or parity reversal, agrees with the continuum action up to $\mathcal{O}\left(a_{t}^{2}, a_{s}^{2}\right)$ terms. The temporal and spatial lattice extents in physical units are given by $a_{t} N_{T}$ and $a_{s} N_{S}$, respectively, so that the only dimensionless combinations consistent with the leading order lattice artefacts are $a_{t}^{2} /\left(a_{t} N_{T}\right)^{2}=1 / N_{T}^{2}$ and $1 / N_{S}^{2}$. Therefore, within perturbation theory, where we cannot dynamically generate additional scales, the leading order lattice artefacts are indistinguishable from $\mathcal{O}\left(1 / N_{T}^{2}, 1 / N_{S}^{2}\right)$ finite size effects.

We choose periodic boundary conditions in time and, to eliminate zero modes and to improve the numerical stability, twisted boundary conditions [20 23] in all spatial directions. The Polyakov line is defined by

$$
L^{(R)}\left(N_{S}, N_{T}\right)=\frac{1}{N_{S}^{3}} \sum_{\mathbf{n}} \frac{1}{d_{R}} \operatorname{tr}\left[\prod_{n_{4}=0}^{N_{T}-1} U_{4}^{R}(n)\right],
$$

where $U_{\mu}^{R}(n) \approx e^{i g A_{\mu}^{R}[(n+1 / 2) a]} \in \mathrm{SU}(3)$ denotes a gauge link in representation $R$, connecting the sites $n$ and $n+\hat{\mu}$, $n_{i} \in\left\{0, \ldots, N_{S}-1\right\}, n_{4} \in\left\{0, \ldots, N_{T}-1\right\}$ and $g=\sqrt{4 \pi \alpha}$. We implement triplet and octet representations $R$ of dimensions $d_{R}=3$ and 8 . The link $U_{4}(n)$ appears within the covariant derivative of the static action $\bar{\psi} D_{4} \psi$, the discretization of which is not unique. We use singly stout-smeared 24] (smearing parameter $\rho=1 / 6$ ) covariant transporters instead of $U_{4}(n)$ as a second, alternative choice, to demonstrate the universality of our findings.

We remark that neither the lattice spacing nor the strong coupling parameter $\alpha$ enter our simulations explicitly. Numerical stochastic perturbation theory (NSPT) [25 27] enables us to directly calculate coefficients of perturbative expansions. We employ the variant of the Langevin algorithm introduced in [28] that only quadratically depends on a time step $\Delta \tau$. Extrapolations to $\Delta \tau=0$ were performed on a subset of lattice volumes where we found agreement within statistical errors between all our extrapolated expansion coefficients and those obtained at $\Delta \tau=0.05$. For the geometries listed in Table I we restrict ourselves to this fixed value, which, within errors, effectively corresponds to $\Delta \tau=0$. 
We expand the logarithm of the smeared and unsmeared Polyakov lines in different representations to obtain the corresponding static energies:

$$
P\left(N_{S}, N_{T}\right)=-\frac{\ln \left\langle L\left(N_{S}, N_{T}\right)\right\rangle}{a N_{T}} \stackrel{N_{S}, N_{T} \rightarrow \infty}{\longrightarrow} \delta m .
$$

Fortunately, the dependence of this logarithm on $N_{T}$ and $N_{S}$ can be deduced and only a few parameters need to be fitted at each order:

$$
\begin{aligned}
a P & =\sum_{n \geq 0}\left[c_{n} \alpha^{n+1}\left(a^{-1}\right)-\frac{f_{n}}{N_{S}} \alpha^{n+1}\left(\left(a N_{S}\right)^{-1}\right)\right. \\
& \left.+\mathcal{O}\left(\frac{1}{N_{T}^{2}}, \frac{1}{N_{S}^{2}}\right)\right] \\
& \approx \sum_{n \geq 0}\left[c_{n}+\Delta_{n}^{(1)}\left(N_{S}\right)+\Delta_{n}^{(2)}\left(N_{S}, N_{T}\right)\right] \alpha^{n+1}\left(a^{-1}\right), \\
\Delta_{n}^{(1)} & =-\frac{1}{N_{S}}\left[f_{n}+\operatorname{logs}_{n}^{(c)}\left(N_{S}\right)\right], \\
\Delta_{n}^{(2)} & =\frac{1}{N_{T}^{2}}\left\{v_{n}-\frac{1}{N_{S}}\left[f_{n}^{(v)}+\operatorname{logs}_{n}^{(v)}\left(N_{S}\right)\right]\right\} \\
& +\frac{1}{N_{S}^{2}}\left\{w_{n}-\frac{1}{N_{S}}\left[f_{n}^{(w)}+\operatorname{logs}_{n}^{(w)}\left(N_{S}\right)\right]\right\} .
\end{aligned}
$$

The $\operatorname{logs} \mathrm{s}_{n}^{(c)}\left(N_{S}\right)$ are polynomials of $\ln \left(N_{S}\right)$ of order $n-1$, with coefficient functions that depend on $f_{j}$ and the $\beta$ function coefficients $\beta_{j}$ where $j \leq n-1$. These terms are entirely determined by the renormalization group running of $\alpha$. The $\operatorname{logs}_{n}^{(v / w)}\left(N_{S}\right)$ are obtained in the same way. In the $N_{T} \rightarrow \infty$ limit $\Delta_{n}^{(1)}$ is the dominant correction while $\Delta_{n}^{(2)}$ includes the leading $\mathcal{O}\left(1 / N_{T}^{2}, 1 / N_{S}^{2}\right)$ lattice artifacts discussed above.

The term $\Delta_{n}^{(1)}$ originates from interactions with mirror images, see also [29]. This effectively produces a static potential between charges separated at distances $a N_{S}$, but without self-energies. Therefore, we expect the high order behaviour of $f_{n}$ and $c_{n}$ to be dominated by one and the same renormalon. This can also be illustrated considering the leading dressed gluon propagator $D(k) \propto$ $1 / k^{2}$, where $k_{4}=0$. With the (formal) ultraviolet cut-off $1 / a$ and an infrared cut-off $1 /\left(a N_{S}\right)$ this can be written as (ignoring lattice corrections),

$$
\begin{aligned}
P & \propto \int_{1 /\left(a N_{S}\right)}^{1 / a} d k k^{2} D(k) \\
& \sim \frac{1}{a} \sum_{n} c_{n} \alpha^{n+1}\left(a^{-1}\right)-\frac{1}{a N_{S}} \sum_{n} c_{n} \alpha^{n+1}\left(\left(a N_{S}\right)^{-1}\right)
\end{aligned}
$$

after perturbatively expanding $D(k)$. When reexpressing $\alpha\left(\left(a N_{S}\right)^{-1}\right)$ in terms of $\alpha\left(a^{-1}\right)$ we may consider two situations:

(a) $N_{S}>e^{n}$. In this limit the last term of Eq. (11) is exponentially suppressed in $n$ and the renormalon can directly be obtained from a large order expansion of $a P$. (b) $N_{S}<e^{n}$. The last term of Eq. (11) is important and the renormalon cancels order-by-order in $n$.

In present-day numerical simulations $N_{S}<e^{n}$, and the term $\Delta_{n}^{(1)}$ needs to be taken into account, in combination with $c_{n}$. A similar phenomenon was numerically observed for the static singlet energy $E(r)=2 m+V(r)$ [19, 30]. This teaches us that to correctly identify the renormalon structure of $\delta m$, it is compulsory to incorporate the $1 / N_{S}$ corrections. So far, in studies of high order perturbative expansions of the plaquette the corresponding finite size terms have been neglected. Our fits indeed yield $f_{n} \simeq c_{n}$ for large $n$, in clear support of the renormalon dominance picture.

In the lattice scheme $\beta_{0}, \beta_{1}$ and $\beta_{2}$ are known [31. The effects of higher $\beta_{j}$ start at $\mathcal{O}\left(\alpha^{5}\right)$, but this uncertainty in our parametrization quickly becomes negligible at high orders where the coefficients $f_{j}$, governed by the $d=1$ renormalon, will dominate. This can be quantified systematically in a large $n$ analysis [32], where any possible renormalon of the lattice $\beta$-function is subleading $(d>1)$. To check this assumption and to justify the truncation at $\beta_{2}$ we have performed fits including $\beta_{j}$ for $j \leq 0,1$ and 2 (see below).

Starting at $\mathcal{O}\left(\alpha^{4}\right)$, one may expect additional finite size terms $\propto \ln \left(N_{T} / N_{S}\right) / N_{S}$ from a possible mixing of the antitriplet interaction between mirror charges with sextet and higher representations, mediated by ultrasoft gluons, in analogy to the mixing of singlet and octet static potentials in potential nonrelativistic QCD (pNRQCD) [33]. These terms are subleading from the renormalon point of view $(d=3)$. Moreover, $a N_{S}$ provides an infrared cut-off to gluon momenta so that one would only expect such contributions in the limit $N_{S} \gg N_{T}$ that we do not investigate and, indeed, we see no numerical evidence of them.

Our data are sensitive to the $1 / N_{T}^{2}$ correction terms within $\Delta_{n}^{(2)}$. However, including $w_{n}$ or $f_{n}^{(w)}$ as additional fit parameters did not significantly improve the $\chi^{2}$-value and so we decided to omit the $1 / N_{S}^{2}$ and $1 / N_{S}^{3}$ terms. Note that these contributions, if present, can numerically easily be distinguished from $1 / N_{S}$ and become irrelevant at relatively small $N_{S}$, unlike $\operatorname{logs}\left(N_{S}\right) / N_{S}$ terms.

As a cross-check we calculate diagrammatically,

$$
\begin{aligned}
c_{0} & =2.1172743570834807985970 \ldots, \\
c_{1} & =11.1425(25), \quad f_{0}=0.76962563284(2), \\
f_{0}^{(w)} & =0.14932(3), \quad w_{0}=v_{0}=f_{0}^{(v)}=0,
\end{aligned}
$$

for the unsmeared Polyakov line. In this case $f_{0}^{(w)}$, the $1 / N_{S}^{3}$ coefficient, does not vanish but it is small. For fundamental sources, $c_{0}$ and $c_{1}$ were known diagrammatically before and $c_{2}$ numerically 29,34 . Our fit reproduces these values. For adjoint sources the above coefficients need to be multiplied by the factor $C_{A} / C_{F}=9 / 4$.

We exemplify the result of our global fit to the unsmeared triplet data obtained on all our geometries (see 


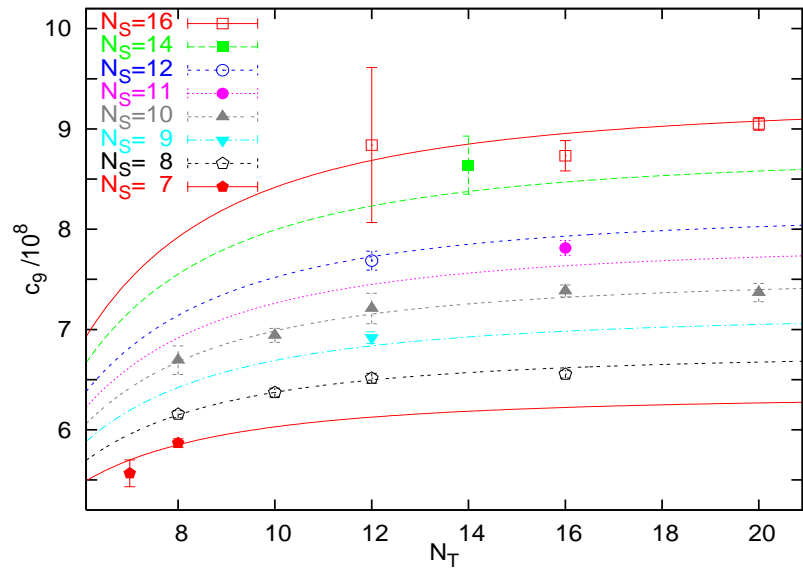

FIG. 1. Comparison between the global fit and data for $n=9$.

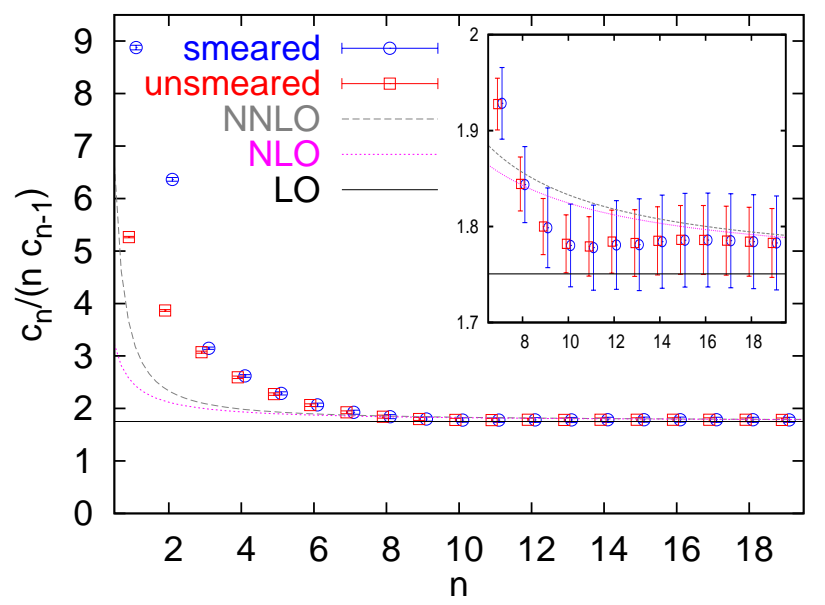

FIG. 2. The ratio $c_{n} /\left(n c_{n-1}\right)$ for the smeared and unsmeared fundamental static self-energies, compared to the prediction Eq. (7) at different orders of the $1 / n$ expansion.

Table I) and orders of perturbation theory (with four parameters per order) in Fig. 1, where a comparison to the $n=9$ data is shown. We find smeared and unsmeared data to be well described by the fits, with reasonable $\chi^{2} / N_{\mathrm{DF}} \approx 1.29$ and 1.46 , respectively. Note that the factorial growth found (and expected) for the coefficients $f_{n}$ produces very sizable $1 / N_{S}$ terms at high orders.

In Fig. 2 we compare the infinite volume extrapolated ratios $c_{n} /\left(n c_{n-1}\right)$ to the theoretical prediction, Eq. (77). LO, NLO and NNLO refer to this prediction, truncated at $\mathcal{O}(1), \mathcal{O}(1 / n)$ and $\mathcal{O}\left(1 / n^{2}\right)$, respectively. The data are robust to subtracting lattice artefacts (the $\Delta^{(2)}$ terms of Eq. (10)) or to truncating at different orders in $\beta_{j}$, see Fig. 3. Particularly reassuring is the universality of the result; fits to smeared and unsmeared Polyakov loop expansions give the same large $n$ behaviour, fully consistent with the dominance and universality of the infrared renormalon; smearing only affects the ultraviolet behaviour. Fits to the octet representation data

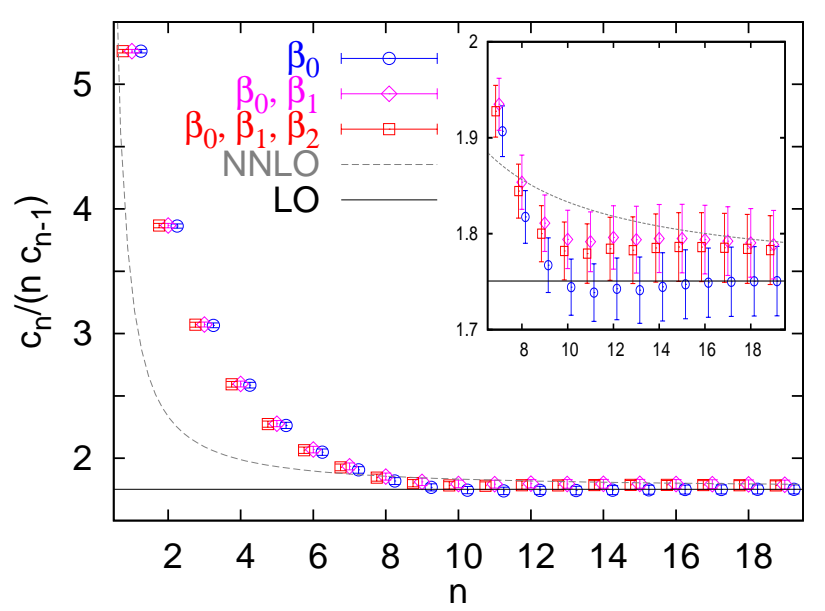

FIG. 3. The same as Fig. 2 for the unsmeared data, truncating at different orders in $\beta_{j}$.

also show exactly the same behaviour, again in agreement with the renormalon dominance picture. Also note that NSPT data for different orders are statistically correlated. These correlations work in our favour. We postpone the details of this to 32].

Finally we determine the normalization of the pole mass renormalon, see Eq. (5), and obtain $N_{m}^{\text {lat }}=$ 18.6(4) for the smeared and $N_{m}^{\text {lat }}=19.0(3)$ for the unsmeared static action. Converting this to the modified minimal subtraction $(\overline{\mathrm{MS}})$ scheme, we find $N_{m}^{\overline{\mathrm{MS}}}=$ $\Lambda_{\text {lat }} N_{m}^{\text {lat }} / \Lambda_{\overline{\mathrm{MS}}}=0.65(2)$. This agrees remarkably well with the estimate $N_{m}^{\overline{\mathrm{MS}}} \simeq 0.62$ of $[30, \underline{35}$. from a $\overline{\mathrm{MS}}$ scheme expansion up to $\mathcal{O}\left(\alpha^{3}\right)$, in support of the claim that renormalon dominance starts at much lower orders in the $\overline{\mathrm{MS}}$ scheme (see for instance [15, 19, 30]). Preliminary results from directly converting our lattice data to the $\overline{\mathrm{MS}}$ scheme further reinforce this claim. Irrespective of the scheme, the heavy quark pole mass can only be defined up to an ambiguity of $\sim 0.65 \Lambda_{\overline{\mathrm{MS}}}$. A more detailed analysis is in preparation [32].

In conclusion, we have obtained the static self-energy of $\mathrm{SU}(3)$ gauge theory in four spacetime dimensions to $\mathcal{O}\left(\alpha^{20}\right)$ in the lattice scheme. For orders $n \gtrsim 9$ we find perfect agreement with the factorial growth of the coefficients, as predicted by the conjectured renormalon picture based on the operator product expansion. Note that this implies that, in the lattice scheme, we expect the renormalon dominance of the plaquette to set in at values $n \gtrsim 4 \times 9$ that so far have not been realized in the literature.

We thank V. Braun, F. Di Renzo, M. García Pérez, H. Perlt, A. Schiller and C. Torrero for discussions. Computations were performed on Regensburg's Athene HPC cluster and at the Leibniz Supercomputing Centre in Munich. C.B. was supported by the Studienstiftung des deutschen Volkes and by the Daimler und Benz Stiftung. This work was supported by DAAD (Acciones Integradas 
Hispano-Alemanas D/07/13355), DFG SFB/TR 55, the EU ITN STRONGnet grant 238353, the Spanish grants FPA2010-16963 and FPA2011-25948, and the Catalan grant SGR2009-00894.

[1] G. 't Hooft, in Proc. Int. School: The whys of subnuclear physics, Erice 1977, edited by A. Zichichi (Plenum, New York, 1978), p. 943.

[2] F. David, Nucl. Phys. B 209, 433 (1982).

[3] I. M. Suslov, J. Exp. Theor. Phys. 100, 1188 (2005) arXiv:hep-ph/0510142.

[4] V. Zakharov, Nucl. Phys. Proc. Suppl. 207, 306 (2010) arXiv:1010.4482 [hep-ph]].

[5] F. Di Renzo, E. Onofri and G. Marchesini, Nucl. Phys. B 457, 202 (1995) arXiv:hep-th/9502095.

[6] G. Burgio, F. Di Renzo, G. Marchesini and E. Onofri, Phys. Lett. B 422, 219 (1998) arXiv:hep-ph/9706209.

[7] R. Horsley, P. E. L. Rakow and G. Schierholz, Nucl. Phys. Proc. Suppl. 106, 870 (2002) arXiv:hep-lat/0110210.

[8] P. E. L. Rakow, PoS LAT2005, 284 (2006) arXiv:hep-lat/0510046.

[9] R. Horsley, G. Hotzel, E. -M. Ilgenfritz, Y. Nakamura, H. Perlt, P. E. L. Rakow, G. Schierholz, A. Schiller, PoS LATTICE2010, 264 (2010) arXiv:1010.4674 [hep-lat]].

[10] M. Beneke, Phys. Rept. 317, 1 (1999) arXiv:hep-ph/9807443.

[11] I. I. Y. Bigi, M. A. Shifman, N. G. Uraltsev and A. I. Vainshtein, Phys. Rev. D 50, 2234 (1994) arXiv:hep-ph/9402360.

[12] M. Beneke and V. M. Braun, Nucl. Phys. B 426, 301 (1994) arXiv:hep-ph/9402364.

[13] K. G. Wilson, Phys. Rev. D 10, 2445 (1974).

[14] M. Beneke, Phys. Lett. B 344, 341 (1995) arXiv:hep-ph/9408380.

[15] A. Pineda, JHEP 0106, $022 \quad$ (2001) arXiv:hep-ph/0105008.
[16] A. Pineda, PhD. thesis "Heavy quarkonium and nonrelativistic effective field theories" (Universitat de Barcelona, 1998).

[17] A. H. Hoang, M. C. Smith, T. Stelzer and S. Willenbrock, Phys. Rev. D 59, 114014 (1999) arXiv:hep-ph/9804227.

[18] M. Beneke, Phys. Lett. B 434, 115 (1998) arXiv:hep-ph/9804241.

[19] G. S. Bali and A. Pineda, Phys. Rev. D 69, 094001 (2004) arXiv:hep-ph/0310130.

[20] G. 't Hooft, Nucl. Phys. B 153, 141 (1979).

[21] G. Parisi, in Proceedings of Progress in Gauge Field Theory, Cargese 1983, edited by G. 't Hooft et al. (Plenum Press, New York, 1984), p. 531.

[22] M. Lüscher and P. Weisz, Nucl. Phys. B 266, 309 (1986).

[23] A. González-Arroyo and C. P. Korthals Altes, Nucl. Phys. B 311, 433 (1988).

[24] C. Morningstar and M. J. Peardon, Phys. Rev. D 69, 054501 (2004) arXiv:hep-lat/0311018.

[25] F. Di Renzo, G. Marchesini, P. Marenzoni and E. Onofri, Nucl. Phys. B Proc. Suppl. 34, 795 (1994).

[26] F. Di Renzo, E. Onofri, G. Marchesini and P. Marenzoni, Nucl. Phys. B 426, 675 (1994) arXiv:hep-lat/9405019.

[27] F. Di Renzo and L. Scorzato, JHEP 0410, 073 (2004) arXiv:hep-lat/0410010.

[28] C. Torrero and G. S. Bali, PoS LATTICE2008, 215 (2008) arXiv:0812.1680 [hep-lat]].

[29] H. D. Trottier, N. H. Shakespeare, G. P. Lepage and P. B. Mackenzie, Phys. Rev. D 65, 094502 (2002) arXiv:hep-lat/0111028.

[30] A. Pineda, J. Phys. G 29, 371 (2003) arXiv:hep-ph/0208031.

[31] A. Bode and H. Panagopoulos, Nucl. Phys. B 625, 198 (2002) arXiv:hep-lat/0110211.

[32] G. S. Bali, C. Bauer, A. Pineda and C. Torrero, in preparation.

[33] N. Brambilla, A. Pineda, J. Soto and A. Vairo, Phys. Rev. D 60, 091502 (1999) arXiv:hep-ph/9903355.

[34] F. Di Renzo and L. Scorzato, JHEP 0102, 020 (2001) arXiv:hep-lat/0012011.

[35] T. Lee, Phys. Rev. D 67, $014020 \quad$ (2003) arXiv:hep-ph/0210032. 